\title{
Helix surfaces in the special linear group
}

\author{
S. Montaldo • I. I. Onnis - A. Passos Passamani
}

Received: 22 January 2014 / Accepted: 16 September 2014 / Published online: 4 October 2014

(C) Fondazione Annali di Matematica Pura ed Applicata and Springer-Verlag Berlin Heidelberg 2014

\begin{abstract}
We characterize helix surfaces (constant angle surfaces) in the special linear group $\operatorname{SL}(2, \mathbb{R})$. In particular, we give an explicit local description of these surfaces by means of a suitable curve and a 1-parameter family of isometries of $\operatorname{SL}(2, \mathbb{R})$.
\end{abstract}

Keywords Special linear group $\cdot$ Helix surfaces $\cdot$ Constant angle surfaces $\cdot$ Homogeneous spaces

Mathematics Subject Classification 53B25

\section{Introduction}

In recent years much work has been done to understand the geometry of surfaces whose unit normal vector field forms a constant angle with a fixed field of directions of the ambient space. These surfaces are called helix surfaces or constant angle surfaces and they have been studied in most of the 3-dimensional geometries. In [2] Cermelli and Di Scala analyzed the case of constant angle surfaces in $\mathbb{R}^{3}$ obtaining a remarkable relation with a Hamilton-Jacobi equation and showing their application to equilibrium configurations of liquid crystals. Later, Dillen-Fastenakels-Van der Veken-Vrancken [4], and Dillen-Munteanu [3], classified the surfaces making a constant angle with the $\mathbb{R}$-direction in the product spaces $\mathbb{S}^{2} \times \mathbb{R}$ and $\mathbb{H}^{2} \times \mathbb{R}$, respectively. Moreover, helix submanifolds have been studied in higher dimensional euclidean spaces and product spaces in $[5,6,10]$.

\footnotetext{
S. Montaldo $(\varangle)$

Dipartimento di Matematica e Informatica, Università degli Studi di Cagliari,

Via Ospedale 72, 09124 Cagliari, Italy

e-mail: montaldo@unica.it
}

I. I. Onnis · A. Passos Passamani

Departamento de Matemática, ICMC, USP, C.P. 668, São Carlos, SP 13560-970, Brasil

e-mail: onnis@icmc.usp.br
A. Passos Passamani
e-mail: apoenapp@icmc.usp.br 
The spaces $\mathbb{S}^{2} \times \mathbb{R}$ and $\mathbb{H}^{2} \times \mathbb{R}$ can be seen as two particular cases of Bianchi-CartanVranceanu spaces (BCV-spaces) which include all 3-dimensional homogeneous metrics whose group of isometries has dimension 4 or 6 , except for those of constant negative sectional curvature. A crucial feature of $\mathrm{BCV}$-spaces is that they admit a Riemannian submersion onto a surface of constant Gaussian curvature, called the Hopf fibration, that, in the cases of $\mathbb{S}^{2} \times \mathbb{R}$ and $\mathbb{H}^{2} \times \mathbb{R}$, it is the natural projection onto the first factor. Consequently, one can consider the angle $\vartheta$ that the unit normal vector field of a surface in a BCV-space forms with the Hopf vector field, which is, by definition, the vector field tangent to the fibers of the Hopf fibration. This angle $\vartheta$ has a crucial role in the study of surfaces in BCV-spaces as shown by Daniel, in [1], where he proved that the equations of Gauss and Codazzi are given in terms of the function $v=\cos \vartheta$ and that this angle is one of the fundamental invariants for a surface in BCV-spaces. Consequently, in [7], the authors considered the surfaces in a $\mathrm{BCV}$-space for which the angle $\vartheta$ is constant, giving a complete local classification in the case that the $\mathrm{BCV}$-space is the Heisenberg space $\mathbb{H}_{3}$.

Later, López-Munteanu, in [8], defined and classified two types of constant angle surfaces in the homogeneous 3-manifold $\mathrm{Sol}_{3}$, whose isometry group has dimension 3. Also, Montaldo-Onnis, in [9], characterized helix surfaces in the 1-parameter family of Berger spheres $\mathbb{S}_{\epsilon}^{3}$, with $\epsilon>0$, proving that, locally, a helix surface is determined by a suitable 1-parameter family of isometries of the Berger sphere and by a geodesic of a 2-torus in the 3-dimensional sphere.

This paper is a continuation of our work [9] and it is devoted to the study and characterization of helix surfaces in the homogeneous 3-manifold given by the special linear group $\operatorname{SL}(2, \mathbb{R})$ endowed with a suitable 1-parameter family $g_{\tau}$ of metrics that we shall describe in sect. 2. Our study of helix surfaces in $\left(\operatorname{SL}(2, \mathbb{R}), g_{\tau}\right)$ will depend on a constant $B:=\left(\tau^{2}+1\right) \cos ^{2} \vartheta-1$, where $\vartheta$ is the constant angle between the normal to the surface and the Hopf vector field of $\operatorname{SL}(2, \mathbb{R})$. A similar constant appeared also in the study of helix surfaces in the Berger sphere (see [9]) but in that case the constant was always positive. Thus we shall divide our study according to the three possibilities: $B>0, B=0$ and $B<0$.

\section{Preliminaries}

Let $\mathbb{R}_{2}^{4}$ denote the 4-dimensional pseudo-Euclidean space endowed with the semi-definite inner product of signature $(2,2)$ given by

$$
\langle v, w\rangle=v_{1} w_{1}+v_{2} w_{2}-v_{3} w_{3}-v_{4} w_{4}, \quad v, w \in \mathbb{R}^{4} .
$$

We identify the special linear group with

$$
\operatorname{SL}(2, \mathbb{R})=\left\{(z, w) \in \mathbb{C}^{2}:|z|^{2}-|w|^{2}=1\right\}=\left\{v \in \mathbb{R}_{2}^{4}:\langle v, v\rangle=1\right\} \subset \mathbb{R}_{2}^{4}
$$

and we shall use the Lorentz model of the hyperbolic plane with constant Gauss curvature -4 , that is

$$
\mathbb{H}^{2}(-4)=\left\{(x, y, z) \in \mathbb{R}_{1}^{3}: x^{2}+y^{2}-z^{2}=-1 / 4\right\},
$$

where $\mathbb{R}_{1}^{3}$ is the Minkowski 3-space. Then the Hopf map $\psi: \operatorname{SL}(2, \mathbb{R}) \rightarrow \mathbb{H}^{2}(-4)$ given by

$$
\psi(z, w)=\frac{1}{2}\left(2 z \bar{w},|z|^{2}+|w|^{2}\right)
$$

is a submersion, with circular fibers, and if we put

$$
X_{1}(z, w)=(i z, i w), \quad X_{2}(z, w)=(i \bar{w}, i \bar{z}), \quad X_{3}(z, w)=(\bar{w}, \bar{z}),
$$


we have that $X_{1}$ is a vertical vector field while $X_{2}, X_{3}$ are horizontal. The vector field $X_{1}$ is called the Hopf vector field.

We shall endow $\operatorname{SL}(2, \mathbb{R})$ with the 1-parameter family of metrics $g_{\tau}, \tau>0$, given by

$$
g_{\tau}\left(X_{i}, X_{j}\right)=\delta_{i j}, \quad g_{\tau}\left(X_{1}, X_{1}\right)=\tau^{2}, \quad g_{\tau}\left(X_{1}, X_{j}\right)=0, \quad i, j \in\{2,3\},
$$

which renders the Hopf map $\psi:\left(\operatorname{SL}(2, \mathbb{R}), g_{\tau}\right) \rightarrow \mathbb{H}^{2}(-4)$ a Riemannian submersion.

For those familiar with the notations of Daniel [1], we point out that $\left(\operatorname{SL}(2, \mathbb{R}), g_{\tau}\right)$ corresponds to a model for a homogeneous space $E(k, \tau)$ with curvature of the basis $k=-4$ and bundle curvature $\tau>0$.

With respect to the inner product in $\mathbb{R}_{2}^{4}$ the metric $g_{\tau}$ is given by

$$
g_{\tau}(X, Y)=-\langle X, Y\rangle+\left(1+\tau^{2}\right)\left\langle X, X_{1}\right\rangle\left\langle Y, X_{1}\right\rangle .
$$

From now on, we denote $\left(\operatorname{SL}(2, \mathbb{R}), g_{\tau}\right)$ with $\operatorname{SL}(2, \mathbb{R})_{\tau}$. Obviously

$$
E_{1}=-\tau^{-1} X_{1}, \quad E_{2}=X_{2}, \quad E_{3}=X_{3},
$$

is an orthonormal basis on $\operatorname{SL}(2, \mathbb{R})_{\tau}$ and the Levi-Civita connection $\nabla^{\tau}$ of $\operatorname{SL}(2, \mathbb{R})_{\tau}$ is given by (see, for example, [11]):

$$
\begin{aligned}
& \nabla_{E_{1}}^{\tau} E_{1}=0, \quad \nabla_{E_{2}}^{\tau} E_{2}=0, \quad \nabla_{E_{3}}^{\tau} E_{3}=0, \\
& \nabla_{E_{1}}^{\tau} E_{2}=-\tau^{-1}\left(2+\tau^{2}\right) E_{3}, \quad \nabla_{E_{1}}^{\tau} E_{3}=\tau^{-1}\left(2+\tau^{2}\right) E_{2}, \\
& \nabla_{E_{2}}^{\tau} E_{1}=-\tau E_{3}, \quad \nabla_{E_{3}}^{\tau} E_{1}=\tau E_{2}, \quad \nabla_{E_{3}}^{\tau} E_{2}=-\tau E_{1}=-\nabla_{E_{2}}^{\tau} E_{3} .
\end{aligned}
$$

Finally, we recall that the isometry group of $\operatorname{SL}(2, \mathbb{R})_{\tau}$ is the 4-dimensional indefinite unitary group $\mathrm{U}_{1}(2)$ that can be identified with:

$$
\mathrm{U}_{1}(2)=\left\{A \in \mathrm{O}_{2}(4): A J_{1}= \pm J_{1} A\right\}
$$

where $J_{1}$ is the complex structure of $\mathbb{R}^{4}$ defined by

$$
J_{1}=\left(\begin{array}{ll}
J & 0 \\
0 & J
\end{array}\right), \quad J=\left(\begin{array}{cc}
0 & -1 \\
1 & 0
\end{array}\right)
$$

while

$$
\mathrm{O}_{2}(4)=\left\{A \in \mathrm{GL}(4, \mathbb{R}): A^{t}=\epsilon A^{-1} \epsilon\right\}, \quad \epsilon=\left(\begin{array}{cc}
I & 0 \\
0 & -I
\end{array}\right), \quad I=\left(\begin{array}{ll}
1 & 0 \\
0 & 1
\end{array}\right)
$$

is the indefinite orthogonal group.

We observe that $\mathrm{O}_{2}(4)$ is the group of $4 \times 4$ real matrices preserving the semi-definite inner product of $\mathbb{R}_{2}^{4}$.

Suppose now we are given a 1-parameter family $A(v), v \in(a, b) \subset \mathbb{R}$, consisting of $4 \times 4$ indefinite orthogonal matrices commuting (anticommuting, respectively) with $J_{1}$. In order to describe explicitly the family $A(v)$, we shall use two product structures of $\mathbb{R}^{4}$, namely

$$
J_{2}=\left(\begin{array}{cccc}
0 & 0 & 0 & 1 \\
0 & 0 & 1 & 0 \\
0 & 1 & 0 & 0 \\
1 & 0 & 0 & 0
\end{array}\right), \quad J_{3}=\left(\begin{array}{cccc}
0 & 0 & 1 & 0 \\
0 & 0 & 0 & -1 \\
1 & 0 & 0 & 0 \\
0 & -1 & 0 & 0
\end{array}\right)
$$

Since $A(v)$ is an indefinite orthogonal matrix, the first row must be a unit vector $\mathbf{r}_{1}(v)$ of $\mathbb{R}_{2}^{4}$ for all $v \in(a, b)$. Thus, without loss of generality, we can take

$$
\begin{aligned}
\mathbf{r}_{1}(v)=( & \cosh \xi_{1}(v) \cos \xi_{2}(v),-\cosh \xi_{1}(v) \sin \xi_{2}(v), \sinh \xi_{1}(v) \cos \xi_{3}(v), \\
& \left.-\sinh \xi_{1}(v) \sin \xi_{3}(v)\right),
\end{aligned}
$$


for some real functions $\xi_{1}, \xi_{2}$ and $\xi_{3}$ defined in $(a, b)$. Since $A(v)$ commutes (anticommutes, respectively) with $J_{1}$ the second row of $A(v)$ must be $\mathbf{r}_{2}(v)= \pm J_{1} \mathbf{r}_{1}(v)$. Now, the four vectors $\left\{\mathbf{r}_{1}, J_{1} \mathbf{r}_{1}, J_{2} \mathbf{r}_{1}, J_{3} \mathbf{r}_{1}\right\}$ form a pseudo-orthonormal basis of $\mathbb{R}_{2}^{4}$, thus the third row $\mathbf{r}_{3}(v)$ of $A(v)$ must be a linear combination of them. Since $\mathbf{r}_{3}(v)$ is unit and it is orthogonal to both $\mathbf{r}_{1}(v)$ and $J_{1} \mathbf{r}_{1}(v)$, there exists a function $\xi(v)$ such that

$$
\mathbf{r}_{3}(v)=\cos \xi(v) J_{2} \mathbf{r}_{1}(v)+\sin \xi(v) J_{3} \mathbf{r}_{1}(v) .
$$

Finally the fourth row of $A(v)$ is $\mathbf{r}_{4}(v)= \pm J_{1} \mathbf{r}_{3}(v)=\mp \cos \xi(v) J_{3} \mathbf{r}_{1}(v) \pm \sin \xi(v) J_{2} \mathbf{r}_{1}(v)$. This means that any 1-parameter family $A(v)$ of $4 \times 4$ indefinite orthogonal matrices commuting (anticommuting, respectively) with $J_{1}$ can be described by four functions $\xi_{1}, \xi_{2}, \xi_{3}$ and $\xi$ as

$$
A\left(\xi, \xi_{1}, \xi_{2}, \xi_{3}\right)(v)=\left(\begin{array}{l}
\mathbf{r}_{1}(v) \\
\pm J_{1} \mathbf{r}_{1}(v) \\
\cos \xi(v) J_{2} \mathbf{r}_{1}(v)+\sin \xi(v) J_{3} \mathbf{r}_{1}(v) \\
\mp \cos \xi(v) J_{3} \mathbf{r}_{1}(v) \pm \sin \xi(v) J_{2} \mathbf{r}_{1}(v)
\end{array}\right)
$$

\section{Constant angle surfaces}

We start this section giving the definition of constant angle surface in $\operatorname{SL}(2, \mathbb{R})_{\tau}$.

Definition 3.1 We say that a surface in the special linear group $\operatorname{SL}(2, \mathbb{R})_{\tau}$ is a helix surface or a constant angle surface if the angle $\vartheta \in[0, \pi)$ between the unit normal vector field and the unit Killing vector field $E_{1}$ (tangent to the fibers of the Hopf fibration) is constant at every point of the surface.

Let $M^{2}$ be an oriented helix surface in $\operatorname{SL}(2, \mathbb{R})_{\tau}$ and let $N$ be a unit normal vector field. Then, by definition,

$$
\left|g_{\tau}\left(E_{1}, N\right)\right|=\cos \vartheta
$$

for fixed $\vartheta \in[0, \pi / 2]$. Note that $\vartheta \neq 0$. In fact, if it were zero then the vector fields $E_{2}$ and $E_{3}$ would be tangent to the surface $M^{2}$, which is absurd since the horizontal distribution of the Hopf map is not integrable. If $\vartheta=\pi / 2$, we have that $E_{1}$ is always tangent to $M$ and, therefore, $M$ is a Hopf cylinder. Therefore, from now on we assume that the constant angle $\vartheta \neq \pi / 2,0$.

The Gauss and Weingarten formulas are

$$
\begin{aligned}
\nabla_{X}^{\tau} Y & =\nabla_{X} Y+\alpha(X, Y), \\
\nabla_{X}^{\tau} N & =-A(X),
\end{aligned}
$$

where with $A$ we have indicated the shape operator of $M$ in $\operatorname{SL}(2, \mathbb{R})_{\tau}$, with $\nabla$ the induced Levi-Civita connection on $M$ and by $\alpha$ the second fundamental form of $M$ in $\operatorname{SL}(2, \mathbb{R})_{\tau}$. Projecting $E_{1}$ onto the tangent plane to $M$ we have

$$
E_{1}=T+\cos \vartheta N
$$

where $T$ is the tangent part which satisfies $g_{\tau}(T, T)=\sin ^{2} \vartheta$.

For all $X \in T M$, we have that

$$
\begin{aligned}
\nabla_{X}^{\tau} E_{1} & =\nabla_{X}^{\tau} T-\cos \vartheta A(X) \\
& =\nabla_{X} T+g_{\tau}(A(X), T) N-\cos \vartheta A(X) .
\end{aligned}
$$


On the other hand, if $X=\sum X_{i} E_{i}$,

$$
\begin{aligned}
\nabla_{X}^{\tau} E_{1} & =\tau\left(X_{3} E_{2}-X_{2} E_{3}\right)=\tau X \wedge E_{1} \\
& =\tau g_{\tau}(J X, T) N-\tau \cos \vartheta J X,
\end{aligned}
$$

where $J X=N \wedge X$ denotes the rotation of angle $\pi / 2$ on $T M$. Identifying the tangent and normal component of (6) and (7) respectively, we obtain

$$
\nabla_{X} T=\cos \vartheta(A(X)-\tau J X)
$$

and

$$
g_{\tau}(A(X)-\tau J X, T)=0 .
$$

Lemma 3.2 Let $M^{2}$ be an oriented helix surface in $\operatorname{SL}(2, \mathbb{R})_{\tau}$ with constant angle $\vartheta$. Then, we have the followings properties.

(i) With respect to the basis $\{T, J T\}$, the matrix associates to the shape operator A takes the form

$$
A=\left(\begin{array}{cc}
0 & -\tau \\
-\tau & \lambda
\end{array}\right)
$$

for some function $\lambda$ on $M$.

(ii) The Levi-Civita connection $\nabla$ of $M$ is given by

$$
\begin{array}{lrl}
\nabla_{T} T=-2 \tau \cos \vartheta J T, & \nabla_{J T} T=\lambda \cos \vartheta J T, \\
\nabla_{T} J T=2 \tau \cos \vartheta T, & \nabla_{J T} J T=-\lambda \cos \vartheta T .
\end{array}
$$

(iii) The Gauss curvature of $M$ is constant and satisfies

$$
K=-4\left(1+\tau^{2}\right) \cos ^{2} \vartheta .
$$

(iv) The function $\lambda$ satisfies the equation

$$
T \lambda+\lambda^{2} \cos \vartheta+4 B \cos \vartheta=0,
$$

where $B:=\left(\tau^{2}+1\right) \cos ^{2} \vartheta-1$.

Proof Point (i) follows directly from (9). From (8) and using

$$
g_{\tau}(T, T)=g_{\tau}(J T, J T)=\sin ^{2} \vartheta, \quad g_{\tau}(T, J T)=0,
$$

we obtain (ii). From the Gauss equation in $\operatorname{SL}(2, \mathbb{R})_{\tau}$ (we refer to the equation in Corollary 3.2 of [1] with $v=\cos \theta$ and $k=-4$ ), and (i), we have that the Gauss curvature of $M$ is given by

$$
\begin{aligned}
K & =\operatorname{det} A+\tau^{2}-4\left(1+\tau^{2}\right) \cos ^{2} \vartheta \\
& =-4\left(1+\tau^{2}\right) \cos ^{2} \vartheta .
\end{aligned}
$$

Finally, (10) follows from the Codazzi equation (see [1]):

$$
\nabla_{X} A(Y)-\nabla_{Y} A(X)-A[X, Y]=-4\left(1+\tau^{2}\right) \cos \vartheta\left(g_{\tau}(Y, T) X-g_{\tau}(X, T) Y\right),
$$

putting $X=T, Y=J T$ and using (ii). In fact, it is easy to check that

$$
\left.-4\left(1+\tau^{2}\right) \cos \vartheta\left(g_{\tau}(J T, T) T-g_{\tau}(T, T) J T\right)\right)=4\left(1+\tau^{2}\right) \cos \vartheta \sin ^{2} \vartheta J T
$$


and

$$
\begin{aligned}
& \nabla_{T} A(J T)-\nabla_{J T} A(T)-A[T, J T] \\
& =\nabla_{T}(-\tau T+\lambda J T)-\nabla_{J T}(-\tau J T)-A(2 \tau \cos \vartheta T-\lambda \cos \vartheta J T) \\
& =\left(4 \tau^{2} \cos \vartheta+T(\lambda)+\lambda^{2} \cos \vartheta\right) J T .
\end{aligned}
$$

As $g_{\tau}\left(E_{1}, N\right)=\cos \vartheta$, there exists a smooth function $\varphi$ on $M$ such that

$$
N=\cos \vartheta E_{1}+\sin \vartheta \cos \varphi E_{2}+\sin \vartheta \sin \varphi E_{3} .
$$

Therefore

$$
T=E_{1}-\cos \vartheta N=\sin \vartheta\left[\sin \vartheta E_{1}-\cos \vartheta \cos \varphi E_{2}-\cos \vartheta \sin \varphi E_{3}\right]
$$

and

$$
J T=\sin \vartheta\left(\sin \varphi E_{2}-\cos \varphi E_{3}\right) .
$$

Also

$$
\begin{aligned}
A(T) & =-\nabla_{T}^{\tau} N=\left(T \varphi-\tau^{-1}\left(2+\tau^{2}\right) \sin ^{2} \vartheta+\tau \cos ^{2} \vartheta\right) J T, \\
A(J T) & =-\nabla_{J T}^{\tau} N=(J T \varphi) J T-\tau T .
\end{aligned}
$$

Comparing (12) with (i) of Lemma 3.2, it results that

$$
\left\{\begin{array}{l}
J T \varphi=\lambda, \\
T \varphi=-2 \tau^{-1} B .
\end{array}\right.
$$

We observe that, as

$$
[T, J T]=\cos \vartheta(2 \tau T-\lambda J T),
$$

the compatibility condition of system (13):

$$
\left(\nabla_{T} J T-\nabla_{J T} T\right) \varphi=[T, J T] \varphi=T(J T \varphi)-J T(T \varphi)
$$

is equivalent to (10).

We now choose local coordinates $(u, v)$ on $M$ such that

$$
\partial_{u}=T .
$$

Also, as $\partial_{v}$ is tangent to $M$, it can be written in the form

$$
\partial_{v}=a T+b J T,
$$

for certain functions $a=a(u, v)$ and $b=b(u, v)$. As

$$
0=\left[\partial_{u}, \partial_{v}\right]=\left(a_{u}+2 \tau b \cos \vartheta\right) T+\left(b_{u}-b \lambda \cos \vartheta\right) J T,
$$

then

$$
\left\{\begin{array}{l}
a_{u}=-2 \tau b \cos \vartheta \\
b_{u}=b \lambda \cos \vartheta
\end{array}\right.
$$

Moreover, the Eq. (10) of Lemma 3.2 can be written as

$$
\lambda_{u}+\cos \vartheta \lambda^{2}+4 B \cos \vartheta=0 .
$$

Depending on the value of $B$, by integration of (17), we have the following three possibilities. 
(i) If $B=0$

$$
\lambda(u, v)=\frac{1}{u \cos \vartheta+\eta(v)},
$$

for some smooth function $\eta$ depending on $v$. Thus the solution of system (16) is given by

$$
\left\{\begin{array}{l}
a(u, v)=-\tau u \cos \vartheta(u \cos \vartheta+2 \eta(v)), \\
b(u, v)=u \cos \vartheta+\eta(v) .
\end{array}\right.
$$

(ii) If $B>0$

$$
\lambda(u, v)=2 \sqrt{B} \tan (\eta(v)-2 \cos \vartheta \sqrt{B} u),
$$

for some smooth function $\eta$ depending on $v$ and system (16) has the solution

$$
\left\{\begin{array}{l}
a(u, v)=\frac{\tau}{\sqrt{B}} \sin (\eta(v)-2 \cos \vartheta \sqrt{B} u), \\
b(u, v)=\cos (\eta(v)-2 \cos \vartheta \sqrt{B} u) .
\end{array}\right.
$$

(iii) If $B<0$

$$
\lambda(u, v)=2 \sqrt{-B} \tanh (\eta(v)+2 \cos \vartheta \sqrt{-B} u),
$$

for some smooth function $\eta$ depending on $v$. Solving the system (16), we have

$$
\left\{\begin{array}{l}
a(u, v)=-\frac{\tau}{\sqrt{-B}} \sinh (\eta(v)+2 \cos \vartheta \sqrt{-B} u), \\
b(u, v)=\cosh (\eta(v)+2 \cos \vartheta \sqrt{-B} u) .
\end{array}\right.
$$

Moreover, in the case (i) the system (13) becomes

$$
\left\{\begin{array}{l}
\varphi_{u}=0 \\
\varphi_{v}=1
\end{array}\right.
$$

and so $\varphi(u, v)=v+c, c \in \mathbb{R}$. In the cases (ii) and (iii), the system (13) becomes

$$
\left\{\begin{array}{l}
\varphi_{u}=-2 \tau^{-1} B \\
\varphi_{v}=0
\end{array}\right.
$$

of which the general solution is given by

$$
\varphi(u, v)=-2 \tau^{-1} B u+c,
$$

where $c$ is a real constant.

With respect to the local coordinates $(u, v)$ chosen above, we have the following characterization of the position vector of a helix surface.

Proposition 3.3 Let $M^{2}$ be a helix surface in $\operatorname{SL}(2, \mathbb{R})_{\tau} \subset \mathbb{R}_{2}^{4}$ with constant angle $\vartheta$. Then, with respect to the local coordinates $(u, v)$ on $M$ defined in (14), the position vector $F$ of $M^{2}$ in $\mathbb{R}_{2}^{4}$ satisfies the following equation:

(a) if $B=0$,

$$
\frac{\partial^{2} F}{\partial u^{2}}=0
$$

(b) if $B \neq 0$,

$$
\frac{\partial^{4} F}{\partial u^{4}}+\left(\tilde{b}^{2}-2 \tilde{a}\right) \frac{\partial^{2} F}{\partial u^{2}}+\tilde{a}^{2} F=0,
$$

where

$$
\tilde{a}=-\tau^{-2} \sin ^{2} \vartheta B, \quad \tilde{b}=-2 \tau^{-1} B .
$$


Proof Let $M^{2}$ be a helix surface and let $F$ be the position vector of $M^{2}$ in $\mathbb{R}_{2}^{4}$. Then, with respect to the local coordinates $(u, v)$ on $M$ defined in (14), we can write $F(u, v)=$ $\left(F_{1}(u, v), \ldots, F_{4}(u, v)\right)$. By definition, taking into account $(11)$, we have that

$$
\begin{aligned}
F_{u} & =\left(\partial_{u} F_{1}, \partial_{u} F_{2}, \partial_{u} F_{3}, \partial_{u} F_{4}\right)=T \\
& =\sin \vartheta\left[\sin \vartheta E_{1 \mid F(u, v)}-\cos \vartheta \cos \varphi E_{2 \mid F(u, v)}-\cos \vartheta \sin \varphi E_{3 \mid F(u, v)}\right] .
\end{aligned}
$$

Using the expression of $E_{1}, E_{2}$ and $E_{3}$ with respect to the coordinates vector fields of $\mathbb{R}_{2}^{4}$, we obtain

$$
\left\{\begin{array}{l}
\partial_{u} F_{1}=\sin \vartheta\left(\tau^{-1} \sin \vartheta F_{2}-\cos \vartheta \cos \varphi F_{4}-\cos \vartheta \sin \varphi F_{3}\right), \\
\partial_{u} F_{2}=-\sin \vartheta\left(\tau^{-1} \sin \vartheta F_{1}+\cos \vartheta \cos \varphi F_{3}-\cos \vartheta \sin \varphi F_{4}\right), \\
\partial_{u} F_{3}=\sin \vartheta\left(\tau^{-1} \sin \vartheta F_{4}-\cos \vartheta \cos \varphi F_{2}-\cos \vartheta \sin \varphi F_{1}\right), \\
\partial_{u} F_{4}=-\sin \vartheta\left(\tau^{-1} \sin \vartheta F_{3}+\cos \vartheta \cos \varphi F_{1}-\cos \vartheta \sin \varphi F_{2}\right) .
\end{array}\right.
$$

Therefore, if $B=0$, taking the derivative of (23) with respect to $u$ and using (18), we obtain that $F_{u u}=0$.

If $B \neq 0$, taking the derivative of (23) with respect to $u$ and using (19), we find two constants $\tilde{a}$ and $\tilde{b}$ such that

$$
\left\{\begin{array}{l}
\left(F_{1}\right)_{u u}=\tilde{a} F_{1}+\tilde{b}\left(F_{2}\right)_{u}, \\
\left(F_{2}\right)_{u u}=\tilde{a} F_{2}-\tilde{b}\left(F_{1}\right)_{u} \\
\left(F_{3}\right)_{u u}=\tilde{a} F_{3}+\tilde{b}\left(F_{4}\right)_{u} \\
\left(F_{4}\right)_{u u}=\tilde{a} F_{4}-\tilde{b}\left(F_{3}\right)_{u},
\end{array}\right.
$$

where

$$
\tilde{a}=\frac{\tau^{-1} \sin ^{2} \vartheta}{2} \varphi_{u}=-\tau^{-2} \sin ^{2} \vartheta B, \quad \tilde{b}=\varphi_{u} .
$$

Finally, taking twice the derivative of (24) with respect to $u$ and using (23) and (24) in the derivative we obtain the desired Eq. (21).

Remark 3.4 As $\langle F, F\rangle=1$, using (21), (23) and (24), we find that the position vector $F(u, v)$ and its derivatives must satisfy the relations:

$$
\begin{array}{lll}
\langle F, F\rangle=1, & \left\langle F_{u}, F_{u}\right\rangle=\tilde{a}, \quad\left\langle F, F_{u}\right\rangle=0, \\
\left\langle F_{u}, F_{\text {uи }}\right\rangle=0, & \left\langle F_{\text {uи }}, F_{\text {uи }}\right\rangle=D, & \left\langle F, F_{\text {uи }}\right\rangle=-\tilde{a}, \\
\left\langle F_{u}, F_{\text {иии }}\right\rangle=-D, & \left\langle F_{\text {ии }}, F_{\text {иии }}\right\rangle=0, & \left\langle F, F_{\text {иии }}\right\rangle=0, \\
\left\langle F_{\text {иии }}, F_{\text {иии }}\right\rangle=E, & &
\end{array}
$$

where

$$
D=\tilde{a} \tilde{b}^{2}-3 \tilde{a}^{2}, \quad E=\left(\tilde{b}^{2}-2 \tilde{a}\right) D-\tilde{a}^{3} .
$$

In addition, as

$$
J_{1} F(u, v)=X_{1 \mid F(u, v)}=-\tau E_{1 \mid F(u, v)}=-\tau\left(F_{u}+\cos \vartheta N\right),
$$

using (21)-(25), we obtain the following identities

$$
\begin{aligned}
& \left\langle J_{1} F, F_{u}\right\rangle=-\tau^{-1} \sin ^{2} \vartheta, \\
& \left\langle J_{1} F, F_{\text {ии }}\right\rangle=0, \\
& \left\langle F_{u}, J_{1} F_{\text {ии }}\right\rangle=\tilde{a}\left(\tilde{b}-\tau^{-1} \sin ^{2} \vartheta\right):=I, \\
& \left\langle J_{1} F_{u}, F_{\text {иии }}\right\rangle=0, \\
& \left\langle J_{1} F_{u}, F_{\text {ии }}\right\rangle+\left\langle J_{1} F, F_{\text {иии }}\right\rangle=0, \\
& \left\langle J_{1} F_{\text {ии }}, F_{\text {иии }}\right\rangle+\left\langle J_{1} F_{u}, F_{\text {ииии }}\right\rangle=0 .
\end{aligned}
$$


Using Remark 3.4 we can prove the following proposition that gives the conditions under which an immersion defines a helix surface.

Proposition 3.5 Let $F: \Omega \rightarrow \operatorname{SL}(2, \mathbb{R})_{\tau} \subset \mathbb{R}_{2}^{4}$ be an immersion from an open set $\Omega \subset \mathbb{R}^{2}$, with local coordinates $(u, v)$, such that the projection of $E_{1}=-\tau^{-1} J_{1} F$ to the tangent space of $F(\Omega) \subset \operatorname{SL}(2, \mathbb{R})_{\tau}$ is $F_{u}$. Then $F(\Omega) \subset \operatorname{SL}(2, \mathbb{R})_{\tau}$ defines a helix surface of constant angle $\vartheta$ if and only if

$$
g_{\tau}\left(F_{u}, F_{u}\right)=g_{\tau}\left(E_{1}, F_{u}\right)=\sin ^{2} \vartheta,
$$

and

$$
g_{\tau}\left(F_{v}, E_{1}\right)-g_{\tau}\left(F_{u}, F_{v}\right)=0 .
$$

Proof Suppose that $F$ is a helix surface of constant angle $\vartheta$. Then

$$
\begin{aligned}
g_{\tau}\left(F_{u}, F_{u}\right) & =-\left\langle F_{u}, F_{u}\right\rangle+\left(1+\tau^{2}\right)\left\langle F_{u}, J_{1} F\right\rangle^{2} \\
& =\tau^{-2} \sin ^{2} \vartheta B+\left(1+\tau^{2}\right)\left(\tau^{-2} \sin ^{4} \vartheta\right) \\
& =\sin ^{2} \vartheta .
\end{aligned}
$$

Similarly

$$
\begin{aligned}
g_{\tau}\left(E_{1}, F_{u}\right) & =\tau^{-1}\left\langle J_{1} F, F_{u}\right\rangle-\tau^{-1}\left(1+\tau^{2}\right)\left\langle J_{1} F, F_{u}\right\rangle\left\langle J_{1} F, J_{1} F\right\rangle \\
& =\tau^{-1}\left\langle J_{1} F, F_{u}\right\rangle\left[1-\left(1+\tau^{2}\right)\right]=\sin ^{2} \vartheta .
\end{aligned}
$$

Finally, using (15), we have

$$
\begin{aligned}
g_{\tau}\left(F_{v}, E_{1}\right)-g_{\tau}\left(F_{u}, F_{v}\right)=- & \frac{a}{\tau} g_{\tau}\left(F_{u}, J_{1} F\right)-\frac{b}{\tau} g_{\tau}\left(J_{1} F_{u}, J_{1} F\right) \\
& -a g_{\tau}\left(F_{u}, F_{u}\right)-b g_{\tau}\left(J_{1} F_{u}, F_{u}\right) \\
= & a \sin ^{2} \vartheta-0-a \sin ^{2} \vartheta-0=0 .
\end{aligned}
$$

For the converse, put

$$
T_{2}=F_{v}-\frac{g_{\tau}\left(F_{v}, F_{u}\right) F_{u}}{g_{\tau}\left(F_{u}, F_{u}\right)}
$$

Then, if we denote by $N$ the unit normal vector field to the surface $F(\Omega),\left\{F_{u}, T_{2}, N\right\}$ is an orthogonal bases of the tangent space of $\operatorname{SL}(2, \mathbb{R})_{\tau}$ along the surface $F(\Omega)$. Now, using (28), we get $g_{\tau}\left(E_{1}, T_{2}\right)=0$, thus $E_{1}=a F_{u}+c N$. Moreover, using (27) and that $g_{\tau}\left(E_{1}, F_{u}\right)=a g_{\tau}\left(F_{u}, F_{u}\right)$, we conclude that $a=1$. Finally,

$$
1=g_{\tau}\left(E_{1}, E_{1}\right)=g_{\tau}\left(F_{u}+c N, F_{u}+c N\right)=\sin ^{2} \vartheta+c^{2},
$$

which implies that $c^{2}=\cos ^{2} \vartheta$. Thus the angle between $E_{1}$ and $N$ is

$$
g_{\tau}\left(E_{1}, N\right)=g_{\tau}\left(F_{u}+\cos \vartheta N, N\right)=\cos \vartheta
$$

\section{The case $B=0$}

Theorem 4.1 Let $M^{2}$ be a helix surface in the $\operatorname{SL}(2, \mathbb{R})_{\tau} \subset \mathbb{R}_{2}^{4}$ with constant angle $\vartheta$ such that $B=0$. Then $\cos \vartheta=\frac{1}{\sqrt{1+\tau^{2}}}$ and, locally, the position vector of $M^{2}$ in $\mathbb{R}_{2}^{4}$, with respect 
to the local coordinates $(u, v)$ on $M$ defined in (14), is given by

$$
F(u, v)=A(v)\left(1,-\frac{\tau u}{1+\tau^{2}}, \frac{\tau u}{1+\tau^{2}}, 0\right),
$$

where $A(v)=A\left(\xi, \xi_{1}, \xi_{2}, \xi_{3}\right)(v)$ is a 1-parameter family of $4 \times 4$ indefinite orthogonal matrices commuting with $J_{1}$, as described in (4), with

$$
\begin{aligned}
& {\left[\xi^{\prime}(v)+\xi_{2}^{\prime}(v)+\xi_{3}^{\prime}(v)\right] \sin \left(\xi_{2}(v)-\xi_{3}(v)\right) \sinh \left(2 \xi_{1}(v)\right)} \\
& -2\left(\xi^{\prime}(v)-\xi_{3}^{\prime}(v)\right) \sinh ^{2} \xi_{1}(v)+2\left[\xi_{1}^{\prime}(v) \cos \left(\xi_{2}(v)-\xi_{3}(v)\right)+\xi_{2}^{\prime}(v) \cosh ^{2} \xi_{1}(v)\right]=0 .
\end{aligned}
$$

\section{Conversely, a parametrization}

$$
F(u, v)=A(v)\left(1,-\frac{\tau u}{1+\tau^{2}}, \frac{\tau u}{1+\tau^{2}}, 0\right),
$$

with $A(v)$ as above, defines a helix surface in the special linear group with constant angle $\vartheta=\arccos \frac{1}{\sqrt{1+\tau^{2}}}$.

Proof Since $B=0$ we have immediately that $\cos ^{2} \vartheta=1 /\left(1+\tau^{2}\right)$. Integrating (20), we obtain that

$$
F(u, v)=h^{1}(v)+u h^{2}(v),
$$

where $h^{i}(v), i=1,2$, are vector fields in $\mathbb{R}_{2}^{4}$, depending only on $v$.

Evaluating in $(0, v)$ the identities:

$$
\begin{aligned}
& \langle F, F\rangle=1, \quad\left\langle F_{u}, F_{u}\right\rangle=0, \\
& \left\langle F, F_{u}\right\rangle=0, \quad\left\langle J_{1} F, F_{u}\right\rangle=-\tau^{-1} \sin ^{2} \vartheta=-\frac{\tau}{1+\tau^{2}},
\end{aligned}
$$

it results that

$$
\begin{aligned}
& \left\langle h^{1}(v), h^{1}(v)\right\rangle=1, \quad\left\langle h^{1}(v), h^{2}(v)\right\rangle=0, \\
& \left\langle h^{2}(v), h^{2}(v)\right\rangle=0, \quad\left\langle J_{1} h^{1}(v), h^{2}(v)\right\rangle=-\frac{\tau}{1+\tau^{2}} .
\end{aligned}
$$

Moreover, using (23) in $(0, v)$, we have that

$$
h^{2}(v)=-\frac{\tau}{1+\tau^{2}}\left(J_{1} h^{1}(v)-h^{3}(v)\right)
$$

where $h^{3}(v)$ is a vector field of $\mathbb{R}_{2}^{4}$ satisfying

$$
\left\langle h^{3}(v), h^{3}(v)\right\rangle=-1, \quad\left\langle h^{1}(v), h^{3}(v)\right\rangle=0, \quad\left\langle J_{1} h^{1}(v), h^{3}(v)\right\rangle=0 .
$$

Consequently, if we fix the orthonormal basis $\left\{\hat{E}_{i}\right\}_{i=1}^{4}$ of $\mathbb{R}_{2}^{4}$ given by

$$
\hat{E}_{1}=(1,0,0,0), \quad \hat{E}_{2}=(0,1,0,0), \quad \hat{E}_{3}=(0,0,1,0), \quad \hat{E}_{4}=(0,0,0,1),
$$

there must exists a 1-parameter family of matrices $A(v) \in \mathrm{O}_{2}(4)$, with $J_{1} A(v)=A(v) J_{1}$, such that

$$
h^{1}(v)=A(v) \hat{E}_{1}, \quad J_{1} h^{1}(v)=A(v) \hat{E}_{2}, \quad h^{3}(v)=A(v) \hat{E}_{3}, \quad J_{1} h^{3}(v)=A(v) \hat{E}_{4} .
$$

Then (31) becomes

$$
F(u, v)=h^{1}(v)-\frac{\tau u}{1+\tau^{2}}\left(J_{1} h^{1}(v)-h^{3}(v)\right)=A(v)\left(1,-\frac{\tau u}{1+\tau^{2}}, \frac{\tau u}{1+\tau^{2}}, 0\right) .
$$


Finally, the 1-parameter family $A(v)$ depends, according to (4), on four functions $\xi_{1}(v), \xi_{2}(v), \xi_{3}(v)$ and $\xi(v)$ and, in this case, condition (28) reduces to $\left\langle F_{u}, F_{v}\right\rangle=0$ which is equivalent to (30).

For the converse, let

$$
F(u, v)=A(v)\left(1,-\frac{\tau u}{1+\tau^{2}}, \frac{\tau u}{1+\tau^{2}}, 0\right),
$$

be a parametrization where $A(v)=A\left(\xi(v), \xi_{1}(v), \xi_{2}(v), \xi_{3}(v)\right)$ is a 1-parameter family of indefinite orthogonal matrices with functions $\xi(v), \xi_{1}(v), \xi_{2}(v), \xi_{3}(v)$ satisfying (30). Since $A(v)$ satisfies (30), then $F$ satisfies (28), thus, in virtue of Proposition 3.5, we only have to show that (27) is satisfied for some constant angle $\vartheta$. For this we put

$$
\gamma(u)=\left(1,-\frac{\tau u}{1+\tau^{2}}, \frac{\tau u}{1+\tau^{2}}, 0\right) .
$$

Now, using (1) and taking into account that $A(v)$ commutes with $J_{1}$, we get

$$
\begin{aligned}
g_{\tau}\left(F_{u}, F_{u}\right) & =-\left\langle A(v) \gamma^{\prime}(u), A(v) \gamma^{\prime}(u)\right\rangle+\left(1+\tau^{2}\right)\left\langle A(v) \gamma^{\prime}(u), J_{1} A(v) \gamma(u)\right\rangle^{2} \\
& =\left(1+\tau^{2}\right)\left\langle\gamma^{\prime}(u), J_{1} \gamma(u)\right\rangle^{2}=\frac{\tau^{2}}{1+\tau^{2}},
\end{aligned}
$$

and we can choose $\vartheta$ such that $\tau^{2} /\left(1+\tau^{2}\right)=\sin ^{2} \vartheta$. Similarly,

$$
\begin{aligned}
g_{\tau}\left(E_{1}, F_{u}\right) & =-\left\langle E_{1}, F_{u}\right\rangle+\left(1+\tau^{2}\right)\left\langle E_{1}, J_{1} F\right\rangle\left\langle F_{u}, J_{1} F\right\rangle \\
& =\frac{\left\langle F_{u}, J_{1} F\right\rangle}{\tau}\left[1-\left(1+\tau^{2}\right)\right] \\
& =(-\tau)\left\langle\gamma^{\prime}(u), J_{1} \gamma(u)\right\rangle=\frac{\tau^{2}}{1+\tau^{2}} .
\end{aligned}
$$

Example 4.2 If we take $\xi_{2}=\xi_{3}=$ constant, (30) becomes $\xi^{\prime}\left(1-\sinh ^{2} \xi_{1}\right)=0$. Thus, if also $\xi=$ constant, we find, from (4), a 1-parameter family $A(v)=A\left(\xi_{1}(v)\right)$ of indefinite orthogonal matrices such that (29) defines a helix surface for any function $\xi_{1}$.

\section{The case $B>0$}

Supposing $B>0$, integrating (21) we have the following

Proposition 5.1 Let $M^{2}$ be a helix surface in $\operatorname{SL}(2, \mathbb{R})_{\tau}$ with constant angle $\vartheta$ so that $B>0$. Then, with respect to the local coordinates $(u, v)$ on $M$ defined in (14), the position vector F of $M^{2}$ in $\mathbb{R}_{2}^{4}$ is given by

$$
F(u, v)=\cos \left(\alpha_{1} u\right) g^{1}(v)+\sin \left(\alpha_{1} u\right) g^{2}(v)+\cos \left(\alpha_{2} u\right) g^{3}(v)+\sin \left(\alpha_{2} u\right) g^{4}(v),
$$

where

$$
\alpha_{1,2}=\frac{1}{\tau}(\tau \sqrt{B} \cos \vartheta \pm B)
$$


are positive real constants, while the $g^{i}(v), i \in\{1, \ldots, 4\}$, are mutually orthogonal vector fields in $\mathbb{R}_{2}^{4}$, depending only on $v$, such that

$$
\begin{aligned}
& g_{11}=\left\langle g^{1}(v), g^{1}(v)\right\rangle=g_{22}=\left\langle g^{2}(v), g^{2}(v)\right\rangle=-\frac{\tau}{2 B} \alpha_{2}, \\
& g_{33}=\left\langle g^{3}(v), g^{3}(v)\right\rangle=g_{44}=\left\langle g^{4}(v), g^{4}(v)\right\rangle=\frac{\tau}{2 B} \alpha_{1} .
\end{aligned}
$$

Proof First, a direct integration of (21), gives the solution

$$
F(u, v)=\cos \left(\alpha_{1} u\right) g^{1}(v)+\sin \left(\alpha_{1} u\right) g^{2}(v)+\cos \left(\alpha_{2} u\right) g^{3}(v)+\sin \left(\alpha_{2} u\right) g^{4}(v),
$$

where

$$
\alpha_{1,2}=\sqrt{\frac{\tilde{b}^{2}-2 \tilde{a} \pm \sqrt{\tilde{b}^{4}-4 \tilde{a} \tilde{b}^{2}}}{2}}
$$

are two constants, while the $g^{i}(v), i \in\{1, \ldots, 4\}$, are vector fields in $\mathbb{R}_{2}^{4}$ which depend only on $v$. Now, taking into account the values of $\tilde{a}$ and $\tilde{b}$ given in (22), we get

$$
\alpha_{1,2}=\frac{1}{\tau}(\tau \sqrt{B} \cos \vartheta \pm B)
$$

Putting $g_{i j}(v)=\left\langle g^{i}(v), g^{j}(v)\right\rangle$, and evaluating the relations (25) in $(0, v)$, we obtain:

$$
\begin{aligned}
& g_{11}+g_{33}+2 g_{13}=1, \\
& \alpha_{1}^{2} g_{22}+\alpha_{2}^{2} g_{44}+2 \alpha_{1} \alpha_{2} g_{24}=\tilde{a}, \\
& \alpha_{1} g_{12}+\alpha_{2} g_{14}+\alpha_{1} g_{23}+\alpha_{2} g_{34}=0, \\
& \alpha_{1}^{3} g_{12}+\alpha_{1} \alpha_{2}^{2} g_{23}+\alpha_{1}^{2} \alpha_{2} g_{14}+\alpha_{2}^{3} g_{34}=0, \\
& \alpha_{1}^{4} g_{11}+\alpha_{2}^{4} g_{33}+2 \alpha_{1}^{2} \alpha_{2}^{2} g_{13}=D, \\
& \alpha_{1}^{2} g_{11}+\alpha_{2}^{2} g_{33}+\left(\alpha_{1}^{2}+\alpha_{2}^{2}\right) g_{13}=\tilde{a}, \\
& \alpha_{1}^{4} g_{22}+\alpha_{1}^{3} \alpha_{2} g_{24}+\alpha_{1} \alpha_{2}^{3} g_{24}+\alpha_{2}^{4} g_{44}=D, \\
& \alpha_{1}^{5} g_{12}+\alpha_{1}^{3} \alpha_{2}^{2} g_{23}+\alpha_{1}^{2} \alpha_{2}^{3} g_{14}+\alpha_{2}^{5} g_{34}=0, \\
& \alpha_{1}^{3} g_{12}+\alpha_{1}^{3} g_{23}+\alpha_{2}^{3} g_{14}+\alpha_{2}^{3} g_{34}=0, \\
& \alpha_{1}^{6} g_{22}+\alpha_{2}^{6} g_{44}+2 \alpha_{1}^{3} \alpha_{2}^{3} g_{24}=E .
\end{aligned}
$$

From (38), (39), (43), (44), it follows that

$$
g_{12}=g_{14}=g_{23}=g_{34}=0 .
$$

Also, from (36), (40) and (41), we obtain

$$
g_{11}=\frac{\tau^{2}\left(D+\alpha_{2}^{4}\right)+2 B \sin ^{2} \vartheta \alpha_{2}^{2}}{\tau^{2}\left(\alpha_{1}^{2}-\alpha_{2}^{2}\right)^{2}}, \quad g_{13}=0, \quad g_{33}=\frac{\tau^{2}\left(D+\alpha_{1}^{4}\right)+2 B \sin ^{2} \vartheta \alpha_{1}^{2}}{\tau^{2}\left(\alpha_{1}^{2}-\alpha_{2}^{2}\right)^{2}} .
$$

Finally, using (37), (42) and (45), we obtain

$$
g_{22}=\frac{\tau^{2}\left(E-2 D \alpha_{2}^{2}\right)-B \sin ^{2} \vartheta \alpha_{2}^{4}}{\tau^{2} \alpha_{1}^{2}\left(\alpha_{1}^{2}-\alpha_{2}^{2}\right)^{2}}, \quad g_{24}=0, \quad g_{44}=\frac{\tau^{2}\left(E-2 D \alpha_{1}^{2}\right)-B \sin ^{2} \vartheta \alpha_{1}^{4}}{\tau^{2} \alpha_{2}^{2}\left(\alpha_{1}^{2}-\alpha_{2}^{2}\right)^{2}} .
$$


We observe that

$$
g_{11}=g_{22}=\frac{\sqrt{B}-\tau \cos \vartheta}{2 \sqrt{B}}<0, \quad g_{33}=g_{44}=\frac{\sqrt{B}+\tau \cos \vartheta}{2 \sqrt{B}}>0 .
$$

Therefore, taking into account (35), we obtain the expressions (34).

We are now in the right position to state the main result of this section.

Theorem 5.2 Let $M^{2}$ be a helix surface in the $\operatorname{SL}(2, \mathbb{R})_{\tau} \subset \mathbb{R}_{2}^{4}$ with constant angle $\vartheta \neq \pi / 2$ so that $B>0$. Then, locally, the position vector of $M^{2}$ in $\mathbb{R}_{2}^{4}$, with respect to the local coordinates $(u, v)$ on $M$ defined in (14), is

$$
F(u, v)=A(v) \gamma(u),
$$

where

$$
\gamma(u)=\left(\sqrt{g_{33}} \cos \left(\alpha_{2} u\right),-\sqrt{g_{33}} \sin \left(\alpha_{2} u\right), \sqrt{-g_{11}} \cos \left(\alpha_{1} u\right), \sqrt{-g_{11}} \sin \left(\alpha_{1} u\right)\right)
$$

is a curve in $\operatorname{SL}(2, \mathbb{R})_{\tau}, g_{11}, g_{33}, \alpha_{1}, \alpha_{2}$ are the four constants given in Proposition 5.1, and $A(v)=A\left(\xi, \xi_{1}, \xi_{2}, \xi_{3}\right)(v)$ is a 1-parameter family of $4 \times 4$ indefinite orthogonal matrices commuting with $J_{1}$, as described in (4), with $\xi=$ constant and

$$
\cosh ^{2}\left(\xi_{1}(v)\right) \xi_{2}^{\prime}(v)+\sinh ^{2}\left(\xi_{1}(v)\right) \xi_{3}^{\prime}(v)=0 .
$$

Conversely, a parametrization $F(u, v)=A(v) \gamma(u)$, with $\gamma(u)$ and $A(v)$ as above, defines a constant angle surface in $\operatorname{SL}(2, \mathbb{R})_{\tau}$ with constant angle $\vartheta \neq \pi / 2$.

Proof With respect to the local coordinates $(u, v)$ on $M$ defined in (14), Proposition 5.1 implies that the position vector of the helix surface in $\mathbb{R}_{2}^{4}$ is given by

$$
F(u, v)=\cos \left(\alpha_{1} u\right) g^{1}(v)+\sin \left(\alpha_{1} u\right) g^{2}(v)+\cos \left(\alpha_{2} u\right) g^{3}(v)+\sin \left(\alpha_{2} u\right) g^{4}(v),
$$

where the vector fields $\left\{g^{i}(v)\right\}_{i=1}^{4}$ are mutually orthogonal and

$$
\begin{aligned}
& \left\|g^{1}(v)\right\|=\left\|g^{2}(v)\right\|=\sqrt{-g_{11}}=\text { constant, } \\
& \left\|g^{3}(v)\right\|=\left\|g^{4}(v)\right\|=\sqrt{g_{33}}=\text { constant. }
\end{aligned}
$$

Thus, if we put $e_{i}(v)=g^{i}(v) /\left\|g^{i}(v)\right\|, i \in\{1, \ldots, 4\}$, we can write:

$$
\begin{aligned}
F(u, v)= & \sqrt{-g_{11}}\left(\cos \left(\alpha_{1} u\right) e_{1}(v)+\sin \left(\alpha_{1} u\right) e_{2}(v)\right) \\
& +\sqrt{g_{33}}\left(\cos \left(\alpha_{2} u\right) e_{3}(v)+\sin \left(\alpha_{2} u\right) e_{4}(v)\right) .
\end{aligned}
$$

Now, the identities (26), evaluated in $(0, v)$, become respectively:

$$
\begin{aligned}
& \alpha_{2} g_{33}\left\langle J_{1} e_{3}, e_{4}\right\rangle-\alpha_{1} g_{11}\left\langle J_{1} e_{1}, e_{2}\right\rangle \\
& \quad+\sqrt{-g_{11} g_{33}}\left(\alpha_{1}\left\langle J_{1} e_{3}, e_{2}\right\rangle+\alpha_{2}\left\langle J_{1} e_{1}, e_{4}\right\rangle\right)=-\tau^{-1} \sin ^{2} \vartheta, \\
& \left\langle J_{1} e_{1}, e_{3}\right\rangle=0, \\
& \alpha_{2}^{3} g_{33}\left\langle J_{1} e_{3}, e_{4}\right\rangle-\alpha_{1}^{3} g_{11}\left\langle J_{1} e_{1}, e_{2}\right\rangle \\
& \quad+\sqrt{-g_{11} g_{33}}\left(\alpha_{1} \alpha_{2}^{2}\left\langle J_{1} e_{3}, e_{2}\right\rangle+\alpha_{1}^{2} \alpha_{2}\left\langle J_{1} e_{1}, e_{4}\right\rangle\right)=-I, \\
& \left\langle J_{1} e_{2}, e_{4}\right\rangle=0, \\
& \alpha_{1}\left\langle J_{1} e_{2}, e_{3}\right\rangle+\alpha_{2}\left\langle J_{1} e_{1}, e_{4}\right\rangle=0, \\
& \alpha_{2}\left\langle J_{1} e_{2}, e_{3}\right\rangle+\alpha_{1}\left\langle J_{1} e_{1}, e_{4}\right\rangle=0 .
\end{aligned}
$$


We point out that to obtain the previous identities we have divided by $\alpha_{1}^{2}-\alpha_{2}^{2}=$ $4 \tau^{-1} \sqrt{B^{3}} \cos \vartheta$ which is, by the assumption on $\vartheta$, always different from zero. From (54) and (55), taking into account the $\alpha_{1}^{2}-\alpha_{2}^{2} \neq 0$, it results that

$$
\left\langle J_{1} e_{3}, e_{2}\right\rangle=0, \quad\left\langle J_{1} e_{1}, e_{4}\right\rangle=0 .
$$

Therefore

$$
\left|\left\langle J_{1} e_{1}, e_{2}\right\rangle\right|=1=\left|\left\langle J_{1} e_{3}, e_{4}\right\rangle\right| .
$$

Substituting (56) in (50) and (52), we obtain the system

$$
\left\{\begin{array}{l}
\alpha_{1} g_{11}\left\langle J_{1} e_{1}, e_{2}\right\rangle-\alpha_{2} g_{33}\left\langle J_{1} e_{3}, e_{4}\right\rangle=\tau^{-1} \sin ^{2} \vartheta \\
\alpha_{1}^{3} g_{11}\left\langle J_{1} e_{1}, e_{2}\right\rangle-\alpha_{2}^{3} g_{33}\left\langle J_{1} e_{3}, e_{4}\right\rangle=I
\end{array}\right.
$$

a solution of which is

$$
\left\langle J_{1} e_{1}, e_{2}\right\rangle=\frac{\tau I-\alpha_{2}^{2} \sin ^{2} \vartheta}{\tau g_{11} \alpha_{1}\left(\alpha_{1}^{2}-\alpha_{2}^{2}\right)}, \quad\left\langle J_{1} e_{3}, e_{4}\right\rangle=\frac{\tau I-\alpha_{1}^{2} \sin ^{2} \vartheta}{\tau g_{33} \alpha_{2}\left(\alpha_{1}^{2}-\alpha_{2}^{2}\right)} .
$$

Now, as

$$
g_{11} g_{33}=-\frac{\sin ^{2} \vartheta}{4 B}, \quad \alpha_{1} \alpha_{2}=\frac{B}{\tau^{2}} \sin ^{2} \vartheta, \quad \alpha_{1}^{2}-\alpha_{2}^{2}=\frac{4 \sqrt{B^{3}}}{\tau} \cos \vartheta,
$$

it results that

$$
\left\langle J_{1} e_{1}, e_{2}\right\rangle\left\langle J_{1} e_{3}, e_{4}\right\rangle=1
$$

Moreover, as

$$
\tau I-\alpha_{2}^{2} \sin ^{2} \vartheta=2 \tau^{-1} \sqrt{B^{3}} \cos \vartheta \sin ^{2} \vartheta
$$

it results that $\left\langle J_{1} e_{1}, e_{2}\right\rangle<0$. Consequently, $\left\langle J_{1} e_{1}, e_{2}\right\rangle=\left\langle J_{1} e_{3}, e_{4}\right\rangle=-1$ and $J_{1} e_{1}=$ $e_{2}, J_{1} e_{3}=-e_{4}$.

Then, if we fix the orthonormal basis of $\mathbb{R}_{2}^{4}$ given by

$$
\tilde{E}_{1}=(0,0,1,0), \quad \tilde{E}_{2}=(0,0,0,1), \quad \tilde{E}_{3}=(1,0,0,0), \quad \tilde{E}_{4}=(0,-1,0,0),
$$

there must exists a 1-parameter family of $4 \times 4$ indefinite orthogonal matrices $A(v) \in \mathrm{O}_{2}(4)$, with $J_{1} A(v)=A(v) J_{1}$, such that $e_{i}(v)=A(v) \tilde{E}_{i}$. Replacing $e_{i}(v)=A(v) \tilde{E}_{i}$ in (49) we obtain

$$
F(u, v)=A(v) \gamma(u)
$$

where

$$
\gamma(u)=\left(\sqrt{g_{33}} \cos \left(\alpha_{2} u\right),-\sqrt{g_{33}} \sin \left(\alpha_{2} u\right), \sqrt{-g_{11}} \cos \left(\alpha_{1} u\right), \sqrt{-g_{11}} \sin \left(\alpha_{1} u\right)\right)
$$

is a curve in $\operatorname{SL}(2, \mathbb{R})_{\tau}$.

Let now examine the 1-parameter family $A(v)$ that, according to (4), depends on four functions $\xi_{1}(v), \xi_{2}(v), \xi_{3}(v)$ and $\xi(v)$. From (15), it results that $\left\langle F_{v}, F_{v}\right\rangle=-\sin ^{2} \vartheta=$ constant. The latter implies that

$$
\frac{\partial}{\partial u}\left\langle F_{v}, F_{v}\right\rangle_{\mid u=0}=0
$$


Now, if we denote by $\mathbf{c}_{1}, \mathbf{c}_{2}, \mathbf{c}_{3}, \mathbf{c}_{4}$ the four colons of $A(v),(57)$ implies that

$$
\left\{\begin{array}{l}
\left\langle\mathbf{c}_{2}{ }^{\prime}, \mathbf{c}_{3}{ }^{\prime}\right\rangle=0 \\
\left\langle\mathbf{c}_{2}^{\prime}, \mathbf{c}_{4}^{\prime}\right\rangle=0
\end{array}\right.
$$

where with ' we means the derivative with respect to $v$. Replacing in (58) the expressions of the $\mathbf{c}_{i}$ 's as functions of $\xi_{1}(v), \xi_{2}(v), \xi_{3}(v)$ and $\xi(v)$, we obtain

$$
\left\{\begin{array}{l}
\xi^{\prime} h(v)=0 \\
\xi^{\prime} k(v)=0
\end{array}\right.
$$

where $h(v)$ and $k(v)$ are two functions such that

$$
h^{2}+k^{2}=4\left(\xi_{1}^{\prime}\right)^{2}+\sinh ^{2}\left(2 \xi_{1}\right)\left(-\xi^{\prime}+\xi_{2}^{\prime}+\xi_{3}^{\prime}\right)^{2} .
$$

From (59) we have two possibilities:

(i) $\xi=$ constant;

or

(ii) $4\left(\xi_{1}^{\prime}\right)^{2}+\sinh ^{2}\left(2 \xi_{1}\right)\left(-\xi^{\prime}+\xi_{2}^{\prime}+\xi_{3}^{\prime}\right)^{2}=0$.

We will show that case (ii) cannot occur, more precisely we will show that if (ii) happens then the parametrization $F(u, v)=A(v) \gamma(u)$ defines a Hopf tube, that is the Hopf vector field $E_{1}$ is tangent to the surface. To this end, we write the unit normal vector field $N$ as

$$
N=\frac{N_{1} E_{1}+N_{2} E_{2}+N_{3} E_{3}}{\sqrt{N_{1}^{2}+N_{2}^{2}+N_{3}^{2}}} .
$$

A long but straightforward computation (that can be also made using a software of symbolic computations) gives

$$
\begin{aligned}
N_{1}= & 1 / 2\left(\alpha_{1}+\alpha_{2}\right) \sqrt{-g_{11}} \sqrt{g_{33}}\left[2 \xi_{1}^{\prime} \cos \left(\alpha_{1} u+\alpha_{2} u-\xi_{2}+\xi_{3}\right)\right. \\
& \left.+\sinh \left(2 \xi_{1}\right) \sin \left(\alpha_{1} u+\alpha_{2} u-\xi_{2}+\xi_{3}\right)\left(-\xi^{\prime}+\xi_{2}^{\prime}+\xi_{3}^{\prime}\right)\right] .
\end{aligned}
$$

Now case (ii) occurs if and only if $\xi_{1}=$ constant $=0$, or if $\xi_{1}=$ constant $\neq 0$ and $-\xi^{\prime}+\xi_{2}^{\prime}+\xi_{3}^{\prime}=0$. In both cases $N_{1}=0$ and this implies that $g_{\tau}\left(N, J_{1} F\right)=-\tau g_{\tau}\left(N, E_{1}\right)=$ 0 , i.e. the Hopf vector field is tangent to the surface. Thus we have proved that $\xi=$ constant.

Finally, in this case, (28) is equivalent to

$$
\tau \cos \vartheta \sqrt{B}\left[\cosh ^{2}\left(\xi_{1}(v)\right) \xi_{2}^{\prime}(v)+\sinh ^{2}\left(\xi_{1}(v)\right) \xi_{3}^{\prime}(v)\right]=0 .
$$

Since $\vartheta \neq \pi / 2$ we conclude that condition (48) is satisfied.

The converse follows immediately from Proposition 3.5 since a direct calculation shows that $g_{\tau}\left(F_{u}, F_{u}\right)=g_{\tau}\left(E_{1}, F_{u}\right)=\sin ^{2} \vartheta$ which is (27), while (48) is equivalent to (28).

\section{The case $B<0$}

In this section we study the case $B<0$. Integrating (21) we have the following result:

Proposition 6.1 Let $M^{2}$ be a helix surface in $\operatorname{SL}(2, \mathbb{R})_{\tau}$ with constant angle $\vartheta$ and $B<0$. Then, with respect to the local coordinates $(u, v)$ on $M$ defined in (14), the position vector F of $M^{2}$ in $\mathbb{R}_{2}^{4}$ is given by 


$$
\begin{aligned}
F(u, v)= & \cos \left(\frac{\tilde{b}}{2} u\right)\left[\cosh (\beta u) w^{1}(v)+\sinh (\beta u) w^{3}(v)\right] \\
& +\sin \left(\frac{\tilde{b}}{2} u\right)\left[\cosh (\beta u) w^{2}(v)+\sinh (\beta u) w^{4}(v)\right],
\end{aligned}
$$

where

$$
\beta=\sqrt{-B} \cos \vartheta
$$

is a real constant, $\tilde{b}=-2 \tau^{-1} B$, while the $w^{i}(v), i \in\{1, \ldots, 4\}$, are vector fields in $\mathbb{R}_{2}^{4}$, depending only on $v$, such that

$$
\begin{aligned}
& \left\langle w^{1}(v), w^{1}(v)\right\rangle=\left\langle w^{2}(v), w^{2}(v)\right\rangle=-\left\langle w^{3}(v), w^{3}(v)\right\rangle=-\left\langle w^{4}(v), w^{4}(v)\right\rangle=1, \\
& \left\langle w^{1}(v), w^{2}(v)\right\rangle=\left\langle w^{1}(v), w^{3}(v)\right\rangle=\left\langle w^{2}(v), w^{4}(v)\right\rangle=\left\langle w^{3}(v), w^{4}(v)\right\rangle=0, \\
& \left\langle w^{1}(v), w^{4}(v)\right\rangle=-\left\langle w^{2}(v), w^{3}(v)\right\rangle=-\frac{2 \beta}{\tilde{b}} .
\end{aligned}
$$

Proof A direct integration of (21), gives the solution

$$
\begin{aligned}
F(u, v)= & \cos \left(\frac{\tilde{b}}{2} u\right)\left[\cosh (\beta u) w^{1}(v)+\sinh (\beta u) w^{3}(v)\right] \\
& +\sin \left(\frac{\tilde{b}}{2} u\right)\left[\cosh (\beta u) w^{2}(v)+\sinh (\beta u) w^{4}(v)\right],
\end{aligned}
$$

where

$$
\beta=\frac{\sqrt{4 \tilde{a}-\tilde{b}^{2}}}{2}=\sqrt{-B} \cos \vartheta
$$

is a constant, while the $w^{i}(v), i \in\{1, \ldots, 4\}$, are vector fields in $\mathbb{R}^{4}$ which depend only on $v$. If $w_{i j}(v):=\left\langle w^{i}(v), w^{j}(v)\right\rangle$, evaluating the relations (25) in $(0, v)$, we obtain

$$
\begin{aligned}
& w_{11}=1 \\
& \frac{\tilde{b}^{2}}{4} w_{22}+\beta^{2} w_{33}+\beta \tilde{b} w_{23}=\tilde{a}, \\
& \frac{\tilde{b}}{2} w_{12}+\beta w_{13}=0, \\
& \frac{\tilde{b}}{2}\left(\beta^{2}-\frac{\tilde{b}^{2}}{4}\right) w_{12}+\beta^{2} \tilde{b} w_{34}+\beta \frac{\tilde{b}^{2}}{2} w_{24}+\beta\left(\beta^{2}-\frac{\tilde{b}^{2}}{4}\right) w_{13}=0, \\
& \left(\beta^{2}-\frac{\tilde{b}^{2}}{4}\right)^{2} w_{11}+\beta^{2} \tilde{b}^{2} w_{44}+2 \beta \tilde{b}\left(\beta^{2}-\frac{\tilde{b}^{2}}{4}\right) w_{14}=D, \\
& \left(\beta^{2}-\frac{\tilde{b}^{2}}{4}\right) w_{11}+\beta \tilde{b} w_{14}=-\tilde{a}, \\
& \frac{\tilde{b}^{2}}{4}\left(3 \beta^{2}-\frac{\tilde{b}^{2}}{4}\right) w_{22}+\beta^{2}\left(\beta^{2}-3 \frac{\tilde{b}^{2}}{4}\right) w_{33}+\beta \frac{\tilde{b}}{2}\left(4 \beta^{2}-\tilde{b}^{2}\right) w_{23}=-D, \\
& \frac{\tilde{b}}{2}\left(3 \beta^{2}-\frac{\tilde{b}^{2}}{4}\right)\left(\beta^{2}-\frac{\tilde{b}^{2}}{4}\right) w_{12}+\tilde{b} \beta^{2}\left(\beta^{2}-3 \frac{\tilde{b}^{2}}{4}\right) w_{34} \\
& +\beta\left(\beta^{2}-3 \frac{\tilde{b}^{2}}{4}\right)\left(\beta^{2}-\frac{\tilde{b}^{2}}{4}\right) w_{13}+\beta \frac{\tilde{b}^{2}}{2}\left(3 \beta^{2}-\frac{\tilde{b}^{2}}{4}\right) w_{24}=0, \\
& \tilde{b}\left(3 \beta^{2}-\frac{\tilde{b}^{2}}{4}\right) w_{12}+\beta\left(\beta^{2}-3 \frac{\tilde{b}^{2}}{4}\right) w_{13}=0, \\
& \frac{2}{2}
\end{aligned}
$$




$$
\begin{aligned}
& \frac{\tilde{b}^{2}}{4}\left(3 \beta^{2}-\frac{\tilde{b}^{2}}{4}\right)^{2} w_{22}+\beta^{2}\left(\beta^{2}-3 \frac{\tilde{b}^{2}}{4}\right)^{2} w_{33} \\
& +\beta \tilde{b}\left(3 \beta^{2}-\frac{\tilde{b}^{2}}{4}\right)\left(\beta^{2}-3 \frac{\tilde{b}^{2}}{4}\right) w_{23}=E .
\end{aligned}
$$

From (62), (66) and (67), it follows that

$$
w_{11}=-w_{44}=1, \quad w_{14}=-\frac{2 \beta}{\tilde{b}} .
$$

Also, from (64) and (70), we obtain

$$
w_{12}=w_{13}=0
$$

and, therefore, from (65) and (69),

$$
w_{24}=w_{34}=0 .
$$

Moreover, using (63), (68) and (71), we get

$$
w_{22}=-w_{33}=1, \quad w_{23}=\frac{2 \beta}{\tilde{b}} .
$$

Theorem 6.2 Let $M^{2}$ be a helix surface in $\operatorname{SL}(2, \mathbb{R})_{\tau}$ with constant angle $\vartheta \neq \pi / 2$ so that $B<0$. Then, locally, the position vector of $M^{2}$ in $\mathbb{R}_{2}^{4}$, with respect to the local coordinates $(u, v)$ on $M$ defined in (14), is given by

$$
F(u, v)=A(v) \gamma(u),
$$

where the curve $\gamma(u)=\left(\gamma_{1}(u), \gamma_{2}(u), \gamma_{3}(u), \gamma_{4}(u)\right)$ is given by

$$
\left\{\begin{array}{l}
\gamma_{1}(u)=\cos \left(\frac{\tilde{b}}{2} u\right) \cosh (\beta u)-\frac{2 \beta}{\tilde{b}} \sin \left(\frac{\tilde{b}}{2} u\right) \sinh (\beta u), \\
\gamma_{2}(u)=\sin \left(\frac{\tilde{b}}{2} u\right) \cosh (\beta u)+\frac{2 \beta}{\tilde{b}} \cos \left(\frac{\tilde{b}}{2} u\right) \sinh (\beta u), \\
\gamma_{3}(u)=\frac{\sin \vartheta}{\sqrt{-B}} \cos \left(\frac{\tilde{b}}{2} u\right) \sinh (\beta u), \\
\gamma_{4}(u)=\frac{\sin \vartheta}{\sqrt{-B}} \sin \left(\frac{\tilde{b}}{2} u\right) \sinh (\beta u),
\end{array}\right.
$$

$\beta=\sqrt{-B} \cos \vartheta, \tilde{b}=-2 \tau^{-1} B$ and $A(v)=A\left(\xi, \xi_{1}, \xi_{2}, \xi_{3}\right)(v)$ is a 1 -parameter family of $4 \times 4$ indefinite orthogonal matrices anticommuting with $J_{1}$, as described in (4), with $\xi=$ constant and

$$
\begin{aligned}
& \sin \vartheta\left[2 \cos \left(\xi_{2}(v)-\xi_{3}(v)\right) \xi_{1}^{\prime}(v)+\left(\xi_{2}^{\prime}(v)+\xi_{3}^{\prime}(v)\right) \sin \left(\xi_{2}(v)-\xi_{3}(v)\right) \sinh \left(2 \xi_{1}(v)\right)\right] \\
& -2 \tau \cos \vartheta\left[\cosh ^{2}\left(\xi_{1}(v)\right) \xi_{2}^{\prime}(v)+\sinh ^{2}\left(\xi_{1}(v)\right) \xi_{3}^{\prime}(v)\right]=0 .
\end{aligned}
$$

Conversely, a parametrization $F(u, v)=A(v) \gamma(u)$, with $\gamma(u)$ and $A(v)$ as above, defines a helix surface in $\operatorname{SL}(2, \mathbb{R})_{\tau}$ with constant angle $\vartheta \neq \pi / 2$.

Proof From (61), we can define the following orthonormal basis in $\mathbb{R}_{2}^{4}$ :

$$
\left\{\begin{array}{l}
e_{1}(v)=w^{1}(v) \\
e_{2}(v)=w^{2}(v) \\
e_{3}(v)=\frac{1}{\sin \vartheta}\left[\sqrt{-B} w^{3}(v)-\tau \cos \vartheta w^{2}(v)\right], \\
e_{4}(v)=\frac{1}{\sin \vartheta}\left[\sqrt{-B} w^{4}(v)+\tau \cos \vartheta w^{1}(v)\right],
\end{array}\right.
$$

with $\left\langle e_{1}, e_{1}\right\rangle=1=\left\langle e_{2}, e_{2}\right\rangle$ and $\left\langle e_{3}, e_{3}\right\rangle=-1=\left\langle e_{4}, e_{4}\right\rangle$. 
Evaluating the identities $(26)$ in $(0, v)$, and taking into account that:

$$
\begin{aligned}
& F(0, v)=w^{1}(v), \\
& F_{u}(0, v)=\frac{\tilde{b}}{2} w^{2}(v)+\beta w^{3}(v), \\
& F_{\text {ии }}(0, v)=\left(\beta^{2}-\frac{\tilde{b}^{2}}{4}\right) w^{1}(v)+\beta \tilde{b} w^{4}(v), \\
& F_{\text {иии }}(0, v)=\frac{\tilde{b}}{2}\left(3 \beta^{2}-\frac{\tilde{b}^{2}}{4}\right) w^{2}(v)+\beta\left(\beta^{2}-\frac{3}{4} \tilde{b}^{2}\right) w^{3}(v), \\
& F_{\text {ииии }}(0, v)=\left(\beta^{4}-\frac{3}{2} \beta^{2} \tilde{b}^{2}+\frac{\tilde{b}^{4}}{16}\right) w^{1}(v)+2 \beta \tilde{b}\left(\beta^{2}-\frac{\tilde{b}^{2}}{4}\right) w^{4}(v),
\end{aligned}
$$

we conclude that

$$
\begin{aligned}
& \left\langle J_{1} w^{3}, w^{4}\right\rangle=-\left\langle J_{1} w^{1}, w^{2}\right\rangle=1, \\
& \left\langle J_{1} w^{3}, w^{2}\right\rangle=\left\langle J_{1} w^{1}, w^{4}\right\rangle=0 \\
& \left\langle J_{1} w^{2}, w^{4}\right\rangle=\left\langle J_{1} w^{1}, w^{3}\right\rangle=-\frac{\tau \cos \vartheta}{\sqrt{-B}} .
\end{aligned}
$$

Then,

$$
\begin{aligned}
&-\left\langle J_{1} e_{1}, e_{2}\right\rangle=\left\langle J_{1} e_{3}, e_{4}\right\rangle=1, \\
&\left\langle J_{1} e_{1}, e_{4}\right\rangle=\left\langle J_{1} e_{1}, e_{3}\right\rangle=\left\langle J_{1} e_{2}, e_{3}\right\rangle=\left\langle J_{1} e_{2}, e_{4}\right\rangle=0 .
\end{aligned}
$$

Therefore, we obtain that

$$
J_{1} e_{1}=-e_{2}, \quad J_{1} e_{3}=-e_{4} .
$$

Consequently, if we consider the orthonormal basis $\left\{\hat{E}_{i}\right\}_{i=1}^{4}$ of $\mathbb{R}_{2}^{4}$ given by

$$
\hat{E}_{1}=(1,0,0,0), \quad \hat{E}_{2}=(0,1,0,0), \quad \hat{E}_{3}=(0,0,1,0), \quad \hat{E}_{4}=(0,0,0,1),
$$

there must exists a 1-parameter family of matrices $A(v) \in \mathrm{O}_{2}(4)$, with $J_{1} A(v)=-A(v) J_{1}$, such that $e_{i}(v)=A(v) \hat{E}_{i}, i \in\{1, \ldots, 4\}$. As

$$
F=\left\langle F, e_{1}\right\rangle e_{1}+\left\langle F, e_{2}\right\rangle e_{2}-\left\langle F, e_{3}\right\rangle e_{3}-\left\langle F, e_{4}\right\rangle e_{4},
$$

computing $\left\langle F, e_{i}\right\rangle$ and substituting $e_{i}(v)=A(v) \hat{E}_{i}$, we obtain that $F(u, v)=A(v) \gamma(u)$, where the curve $\gamma(u)$ of $\operatorname{SL}(2, \mathbb{R})_{\tau}$ is given in (72).

Let now examine the 1-parameter family $A(v)$ that, according to (4), depends on four functions $\xi_{1}(v), \xi_{2}(v), \xi_{3}(v)$ and $\xi(v)$. Similarly to what we have done in the proof of Theorem 5.2 we have that the condition

$$
\frac{\partial}{\partial u}\left\langle F_{v}, F_{v}\right\rangle_{\mid u=0}=0
$$

implies that the functions $\xi_{1}(v), \xi_{2}(v), \xi_{3}(v)$ and $\xi(v)$ satisfy the equation

$$
\xi^{\prime}\left[2 \sin \left(\xi_{2}-\xi_{3}\right) \xi_{1}^{\prime}-\left(\xi_{2}^{\prime}+\xi_{3}^{\prime}-\xi^{\prime}\right) \cos \left(\xi_{2}-\xi_{3}\right) \sinh \left(2 \xi_{1}\right)\right]=0 .
$$

Then we have two possibilities:

(i) $\xi=$ constant;

or

(ii) $2 \sin \left(\xi_{2}-\xi_{3}\right) \xi_{1}^{\prime}-\left(\xi_{2}^{\prime}+\xi_{3}^{\prime}-\xi^{\prime}\right) \cos \left(\xi_{2}-\xi_{3}\right) \sinh \left(2 \xi_{1}\right)=0$. 
Also in this case, using the same argument as in Theorem 5.2, condition (ii) would implies that the surface is a Hopf tube, thus we can assume that $\xi=$ constant.

Finally, a long but straightforward computation shows that, in the case $\xi=$ constant, (28) is equivalent to (73).

The converse of the theorem follows immediately from Proposition 3.5 since a direct calculation shows that $g_{\tau}\left(F_{u}, F_{u}\right)=g_{\tau}\left(E_{1}, F_{u}\right)=\sin ^{2} \vartheta$ which is (27) while (73) is equivalent to (28).

Acknowledgments S. Montaldo was supported by P.R.I.N. 2010/11-Varietà reali e complesse: geometria, topologia e analisi armonica-Italy and INdAM. A. Passos Passamani was supported by Capes—Brazil.

\section{References}

1. Daniel, B.: Isometric immersions into 3-dimensional homogeneous manifolds. Comment. Math. Helv. 82, 87-131 (2007)

2. Cermelli, P., Di Scala, A.J.: Constant-angle surfaces in liquid crystals. Phil. Mag. 87, 1871-1888 (2007)

3. Dillen, F., Munteanu, M.I.: Constant angle surfaces in $\mathbb{H}^{2} \times \mathbb{R}$. Bull. Braz. Math. Soc. (N.S.) 40, $85-97$ (2009)

4. Dillen, F., Fastenakels, J., Van der Veken, J., Vrancken, L.: Constant angle surfaces in $\mathbb{S}^{2} \times \mathbb{R}$. Monatsh. Math. 152, 89-96 (2007)

5. Di Scala, A., Ruiz-Hernández, G.: Higher codimensional Euclidean helix submanifolds. Kodai Math. J. 33, 192-210 (2010)

6. Di Scala, A., Ruiz-Hernández, G.: Helix submanifolds of Euclidean spaces. Monatsh. Math. 157, 205-215 (2009)

7. Fastenakels, J., Munteanu, M.I., Van Der Veken, J.: Constant angle surfaces in the Heisenberg group. Acta Math. Sin. (Engl. Ser.) 27, 747-756 (2011)

8. López, R., Munteanu, M.I.: On the geometry of constant angle surfaces in $\mathrm{Sol}_{3}$. Kyushu J. Math. 65, 237-249 (2011)

9. Montaldo, S., Onnis, I.I.: Helix surfaces in the Berger sphere. Israel J. Math. (2014). doi:10.1007/ s11856-014-1055-6

10. Ruiz-Hernández, G.: Minimal helix surfaces in $N^{n} \times \mathbb{R}$. Abh. Math. Semin. Univ. Hambg. 81, 55-67 (2011)

11. Torralbo, F.: Rotationally invariant constant mean curvature surfaces in homogeneous 3 -manifolds. Differ. Geom. Appl. 28, 593-607 (2010) 